\title{
Control de riego en cultivos de ahuyama en Sincelejo, Sucre (Colombia) gestionados a través del Internet de las Cosas
}

\author{
Carlos S. Cohen-Manrique ${ }^{\star}$, Andrea F. Burbano-Bustos, Rodrigo D. Salgado-Ordosgoitia y Rafael H. Merlano-Porto \\ Corporación Universitaria del Caribe, Facultad de Ciencias Básicas, Ingeniería y Arquitectura, Sincelejo, Colombia. \\ (correo-e: carlos.cohen@cecar.edu.co, andrea.burbano@cecar.edu.co,rodrigo.salgado@cecar.edu.co, \\ rafael.merlano@cecar.edu.co)
}

* Autor a quien debe ser dirigida la correspondencia

Recibido Mar. 11, 2020; Aceptado May.12, 2020; Versión final Jul. 16, 2020, Publicado Oct. 2020

\begin{abstract}
Resumen
Se plantea un sistema de riego automático para cultivos de ahuyama en el departamento de Sucre, Colombia. El sistema diseñado se compone de un controlador proporcional, integral y derivativo (PID) sintonizado y un sistema de adquisición de datos conectado a la nube mediante herramientas del Internet de las Cosas. EI usuario controla el cultivo de manera remota y gestiona la información histórica y en tiempo real para evaluar el requerimiento hídrico del cultivo de variables atmosféricas y suelo. Los valores referenciales obtenidos de las diferencias entre la humedad deseada en el cultivo y la humedad medida en tiempo real en el suelo y el microclima del cultivo fueron inferiores al $2 \%$. El sistema demostró ser eficiente, estable y optimiza el error en estado estacionario, lo que contribuye para la implementación en campo a bajo costo y resultando en un aporte importante para la región.
\end{abstract}

Palabras clave: controlador PID; sistemas de riego; internet de las cosas; agricultura de precisión

\section{Irrigation control in ahuyama crops in Sincelejo, Sucre (Colombia) managed through the Internet of Things (IoT)}

\begin{abstract}
An automatic irrigation system for ahuyama crops is proposed in the department of Sucre, Colombia. The designed system is composed of a tuned proportional, integral, and derivative controller (PID) and of a data acquisition system connected to a cloud using Internet of Things tools. The user controls the crop remotely and manages the historical and in real time information to assess the water requirement of the crop according to atmospheric and soil variables. The reference values obtained from the differences between the desired humidity in the crop and the humidity measured in real time in the soil and the microclimate of the crop were lower than $2 \%$. The system proved to be efficient, stable, and optimizes the error in steady state, which contributes to low-cost field implementation and makes the system an important contribution to the region.
\end{abstract}

Keywords: PID controller; irrigation systems; internet of things; precision agriculture 


\section{INTRODUCCIÓN}

Debido a las múltiples dificultades climatológicas, económicas y políticas que soporta la región Sabanas del departamento de Sucre en cuanto a la sostenibilidad de sus reservorios hídricos y su correcta administración, se ha estudiado desde hace varios años diferentes estrategias tecnológicas y agroindustriales orientadas hacia la optimización de éste recurso natural necesario para las diferentes actividades productivas y para el sostenimiento social en la región Sabanas, valles del río Sinú, los Montes de María y sus alrededores, orientadas a la optimización del recurso hídrico y su potencialización agrícola (Liu y Kwaku, 2018; CohenManrique et al., 2019). Todas ellas diseñadas, modeladas e implementadas en sistemas de riego por goteo, por aspersión, por fertirriego, entre otras; minimizando el impacto climatológico natural que se produce en los cultivos debidos a los síntomas de estrés hídrico producidos por los altos índices de radiación solar, altas temperaturas y pocas posibilidades pluviométricas, que impactan en los cultivos en términos productivos y sociales.

En el documento se presentan los aspectos más importantes relacionados con una plantación de ahuyama apoyados por un sistema de control para riego automático, basado en un controlador PID básico, y modelado en Matlab a través de ecuaciones relacionadas con el requerimiento hídrico en el suelo y en la atmosfera. Los cultivos de ahuyama estuvieron ubicados en la ciudad de Sincelejo, Sucre (Colombia) situada aproximadamente a unos 213 metros sobre el nivel del mar, y que cuenta con un clima cálido oscilante entre los 27 y los 38 grados centígrados. Sus principales fuentes de desarrollo son la ganadería y la agricultura, y ambos sectores económicos han sido golpeados en los últimos años debido a los fuertes efectos del cambio climático, a la escasa pluviosidad y sobre todo por los precarios sistemas tecnológicos utilizados para la administración del agua. Los aspectos mencionados han impactado negativamente disminuyendo notablemente la producción en ambas áreas, reduciendo considerablemente las oportunidades competitivas de sus productos en relación con otras regiones del país y del mundo. Lo cual implica que se deben fomentar e implementar más y mayores proyectos de desarrollo e impacto social y económico orientados a la optimización de recursos en el campo agroalimentario.

Un dato importante que explica las dificultades relacionadas con el clima y los cultivos en el departamento de Sucre, está relacionado con los excedentes y/o el déficit de aguas que se presentan en sus cuencas de referencia, esto es el índice de aridez del suelo, que en buena parte de la región se encuentra en el rango entre 0,40 y 0,49; lo que según la escala de Knoche (IAK) define dicha región en el intervalo de la aridez severa, o así mismo, según la clasificación formulada por Martonne, en terrenos semiáridos (Lozano-Rivas, 2018). En la ecuación 1 se presenta el modelo del IAK utilizado para el cultivo, donde P es el índice de precipitación media anual medida en milímetros $(\mathrm{mm})$, T es la temperatura media anual medida en grados centígrados $\left({ }^{\circ} \mathrm{C}\right)$ y $\mathrm{n}$ es el número medio de días de lluvia al año.

$$
I_{x}=\frac{n P}{100(T+10)}
$$

Los valores obtenidos mediante estos índices definen en buena parte la dificultad y las limitaciones del ejercicio agrícola en esta región de Colombia; y en manera particular, el cultivo de ahuyama, hortaliza tradicional en el país y que ocupa una posición importante en su producción y consumo. Esta hortaliza de naturaleza oblonga y de altos contenidos vitamínicos ha trascendido históricamente de modo que reviste una gran importancia en el campesinado y la industria agroalimentaria de la región y del país ya que es la base de productos de consumo diario, tales como harinas, almidones y concentrados para animales (Pinilla, 2017). Así mismo, para el consumo humano se prepara en cremas, sopas, cocida, dulces, entre otras presentaciones. (Marsiglia Fuentes et al., 2018).

\section{OTROS ANTECEDENTES}

La producción de ahuyama ha venido recuperándose en los últimos años, tanto en el departamento de Sucre (Figura 1a) como en todo el país (Figura 1b), esto debido a la implementación de mejores políticas e incentivos agrícolas para las hortalizas (Orozco et al., 2019). En la figura se puede observar el incremento en su producción de al menos un 160\% entre los años 2016 a 2018 en el departamento de Sucre. Y un aumento aproximado de al menos un $18 \%$ a nivel nacional. Datos que confirman la importancia de este producto en la región.

Con todo lo anterior, se demuestra la importancia del proyecto aplicado, en el cual se sistematiza en tiempo real las principales variables relacionadas con los niveles de consumo de agua para riegos en los cultivos de ahuyamas en el departamento de Sucre, utilizando estrategias tecnológicas conexas al internet de las cosas y al control automático de los procesos; a fin de generar un impacto social, económico y ambiental que permitiese la sostenibilidad en esas tres áreas; para garantizar la producción de dichos cultivos, con uso óptimo de los recursos naturales. En otras palabras, se buscó aprovechar debidamente los recursos hídricos y terrestres para garantizar con efectividad la cosecha de ahuyamas en el departamento de Sucre, mediante 
un control de riego diseñado para mitigar los efectos del cambio climático que amenacen la producción de ahuyamas y optimizar los consumos energéticos e hídricos. Entregando a la comunidad científica un sistema de control automático e innovador para riego por aspersión en cultivos de ahuyama en el departamento de Sucre, diseño que resulta ser creativo y sustentable porque tecnifica a la agricultura dado que, dota a los agricultores de nuevas tecnologías y herramientas que permiten fácil y sosteniblemente el ejercicio de la agricultura.

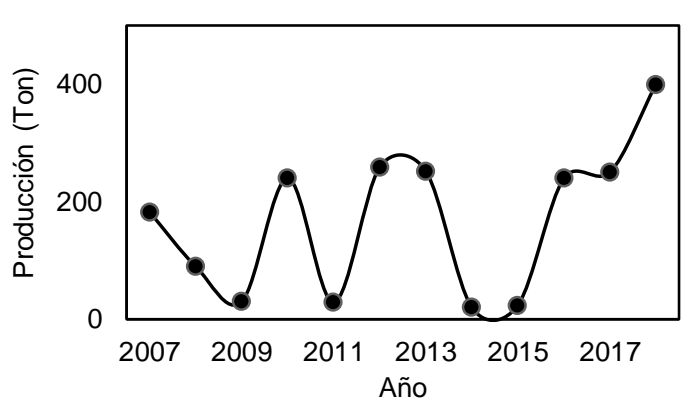

a.

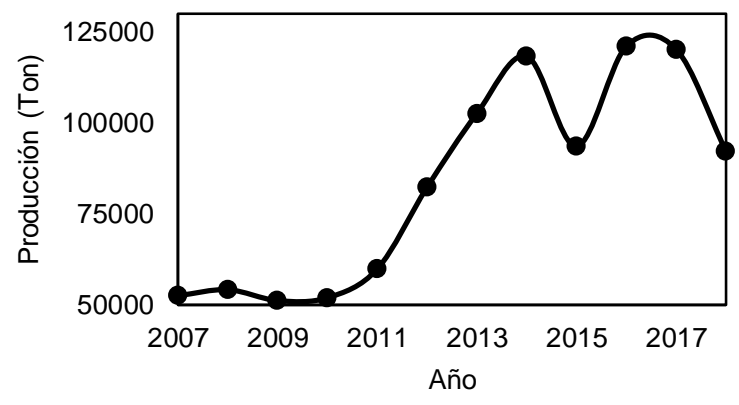

b.

Fig. 1: Datos de producción de ahuyama en el departamento de Sucre (1a) y en Colombia (1b).

Es importante tener en cuenta la importancia de los sistemas de riego para la región Caribe, considerando por ejemplo que las zonas cultivables del departamento de Sucre que reciben altas descargas relacionadas con radiación solar y muy poca contribución hídrica conexa con precipitaciones (Domínguez-Pérez y MercadoFernández, 2020), solo durante cortos períodos de tiempo el nivel pluviométrico aumenta. Razones importantes a tener en cuenta a la hora de elegir una alternativa tecnológica viable para sistemas de riego que garanticen un saludable crecimiento de los cultivos. Específicamente en la Figura 2 se muestra la tasa de precipitación máxima mensual (en promedio) registrada durante el año 2019 en la estación meteorológica EI Paraíso, aeropuerto Rafael Bravo de Corozal - Sucre, registrado por el IDEAM con el código 25025080 y ubicada a $9^{\circ} 20^{\prime}$ de latitud norte, $75^{\circ} 17^{\prime}$ longitud oeste y una elevación de 166 metros sobre el nivel del mar.

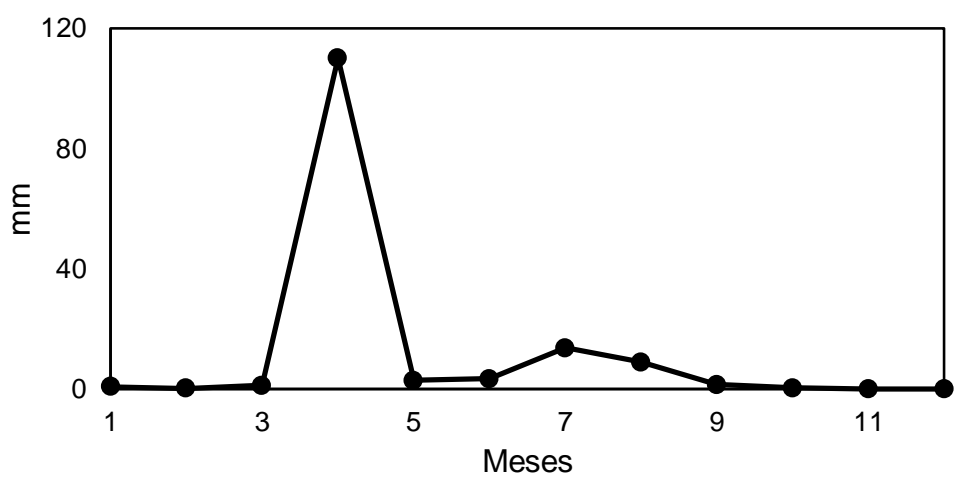

Fig. 2: Precipitación promedio obtenida durante los meses de la siembra y cosecha de la ahuyama en la región de las Sabanas del departamento de Sucre, estación meteorológica El Paraíso, aeropuerto Rafael Bravo.

Evidentemente, en esta Figura 2 se puede observar que la concentración de las lluvias se registra durante los meses en los que se acostumbra a realizar los cultivos de ahuyama en la región de las Sabanas del departamento de Sucre (González et al., 2019). De este modo se puede afirmar que el mes propicio para la siembra por lo general es el mes de marzo, de modo que en el crecimiento de las plántulas sea de máximo provecho debido a las altas cantidades de líquido que se producen durante el mes de abril. Durante los meses mayo y junio la labor de los sistemas de riego recobra importancia debido a la disminución pronunciada de las precipitaciones.

\section{METODOLOGIA}

Metodológicamente, se realizó el diseño e implementación de un sistema de control de riego para cultivos de ahuyama, basado en modelos del microclima de la planta de ahuyama ajustada a los terrenos de la sabana del departamento de Sucre (Colombia), utilizando datos adquiridos en tiempo real a través de una estación meteorológica y modelos matemáticos aplicados a la simulación de la reacción del suelo y subsuelo de la región. Seguidamente se utilizan dichos datos en tiempo real, para alimentar un sistema de adquisición de datos y controlador PID para la optimización del recurso hídrico en el sembrado, a través de conexiones sensóricas inalámbricas y el registro permanente de los datos en la nube para futuros modelamientos a través de la ciencia de datos. A continuación, se explica con mayor detalle cada uno de las fases implementadas en el estudio. 


\section{Sistema de Adquisición de Datos y Controlador}

El sistema de riego automático se diseñó a partir de una aplicación de control proporcional, integral y derivativo básico orientado a controlar los niveles de error presentado al momento de medir los requerimientos hídricos teóricos de una plantación en base a modelos matemáticos que explican el comportamiento de la evapotranspiración, mediante el modelo de Penman-Monteith, el crecimiento de la raíz del cultivo, sus etapas fenológicas, las características intrínsecas del suelo cultivable de la región, la tasa de infiltración, las láminas acumuladas de agua debida a la escorrentía, entre tantas otras variables que se definen en (Cohen-Manrique et al., 2018) contrastadas con los requerimientos reales del mismo, medibles a través de datos en tiempo real capturados por una estación meteorológica, dispositivos sensóricos inalámbricos y dispositivos electrónicos para la adquisición, almacenamiento en la nube y control de datos; además de electroválvulas calibradas y configuradas para optimizar el consumo energético e hídrico del sistema de riego en cultivos de ahuyama. Estos datos, tomados en tiempo real, se utilizaron para establecer comportamientos y en un futuro plantear sistemas de control predictivo para el riego. En la Figura 3 se muestra el esquema del sistema implementado.

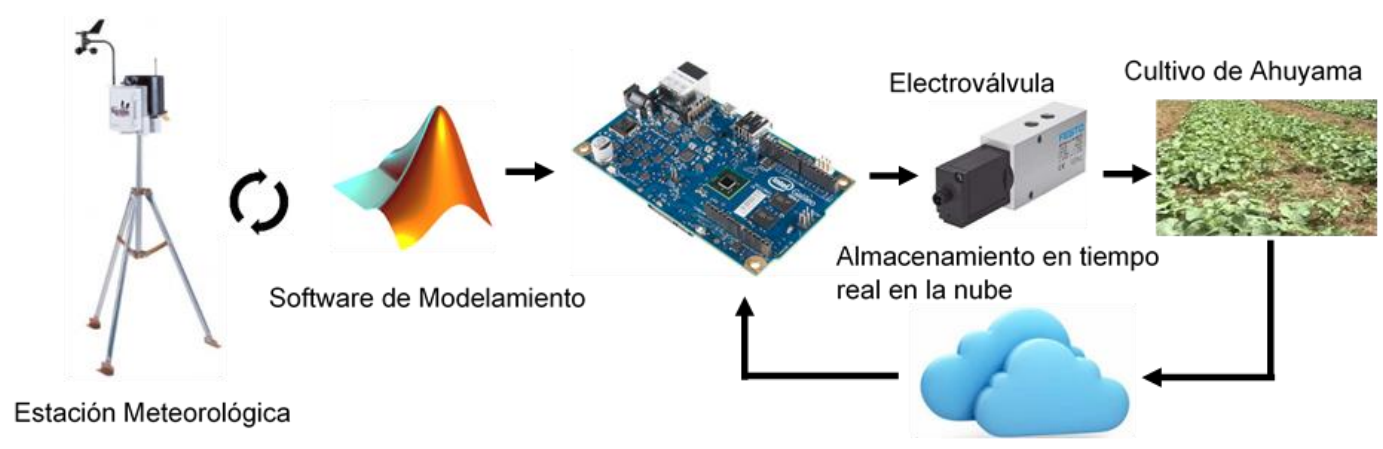

Fig. 3: Diagrama de bloques del sistema de control utilizado para el control de riego de ahuyama.

El sistema emplea modelamiento matemático utilizando ecuaciones que describen la infiltración del agua en el suelo (Kirkham, 2014), la escorrentía (Hernández, 2010), el crecimiento temporal de las raíces de las plantas (Castellaro y Squella, 2006), la evapotranspiración del cultivo (modelo de Penman Monteith) y todas las variables que componen el microclima de las plantas de ahuyama, ajustando su comportamiento a los parámetros específicos de la región de evaluación y a los del cultivo, definidos por el coeficiente de cultivo Kc y evapotranspiración de cultivo ETo (Allen, 2006). En cuanto al suelo, se utilizaron los modelos planteados en (Cohen-Manrique et al., 2018) que describen la capacidad de campo del suelo y su densidad aparente en términos del porcentaje de humedad $(\% \mathrm{H})$ in situ, según el tipo de suelo, que para la región de siembra fueron determinados como arcilloso, franco y franco arcilloso. Además de sensores de humedad del suelo (HD-38) con sonda, y el sensor RT1 para medir la temperatura al nivel del suelo, con intervalos de medida de 20 segundos por muestra.

Luego de capturar las variables que alimentan el modelo, se utilizó tarjeta de adquisición de datos Intel Galileo® Gen 2, de manera que se optimizaran las cantidades específicas de líquido suministrada al cultivo a través de las electroválvulas controladas en tiempo real y almacenando directamente la información en la nube, a través de la plataforma libre ThinkSpeak. En cuanto al actuador (electroválvula), se utilizó una válvula tipo proporcional MPYE-5-1/8 de FESTO con sensor de posición, con un rango de operación entre los 0 a 5 voltios para su máximo y mínimo caudal respectivamente, así mismo los sensores inalámbricos y estación meteorológica WatchDog Modelo 2900ET descrita en (Cohen-Manrique et al., 2019) formando una red inalámbrica de sensores (WSAN) administrada por la tarjeta de control. El objetivo del software y de los sistemas de adquisición de datos fue el de advertir regularmente al usuario a través de sensores el estado actual de la red, censando y cronometrando la información del suelo y del ambiente, para luego cargarla en los nodos de enrutamiento inalámbrico (García et al., 2020).

\section{Tipo de cultivo}

El cultivo de ahuyama se adapta de manera sencilla a las características del suelo en la región, con requerimientos líquidos aproximados de $5 \mathrm{~mm} /$ día de agua, habituada a los suelos francos y arcillosos (tal como los utilizados en el modelado antes descrito), con una variación en su pH entre un 5,80 a 6,80 (Çelekli et al., 2019). En cuanto a su crecimiento y su relación de consumo líquido anaeróbico, en la Figura 4 se muestra la relación práctica entre el tiempo de crecimiento del cultivo y el coeficiente Kc de este, el cual representa el consumo de agua de la plantación debida al estrés producto del calor, además de las condiciones meteorológicas y ambientales a las que está sujeto el cultivo a campo abierto. El tiempo de crecimiento de la ahuyama en los cultivos de la región, es de aproximadamente 12 semanas ( 3 meses), con un rendimiento aproximado de 11,3 toneladas por hectárea en el 2017 (Rodríguez et al., 2018). En la Figura 
5 se muestran las diferentes etapas del cultivo de ahuyama durante el tiempo de siembra, esto es, germinación y nacimiento, crecimiento (Einicial), maduración ( $E_{\text {desarrollo }}$ y y cosecha ( $\left.E_{\text {fin }}\right)$ así mismo los respectivos valores de Kc (coeficiente de cultivo) en cada una de las etapas.

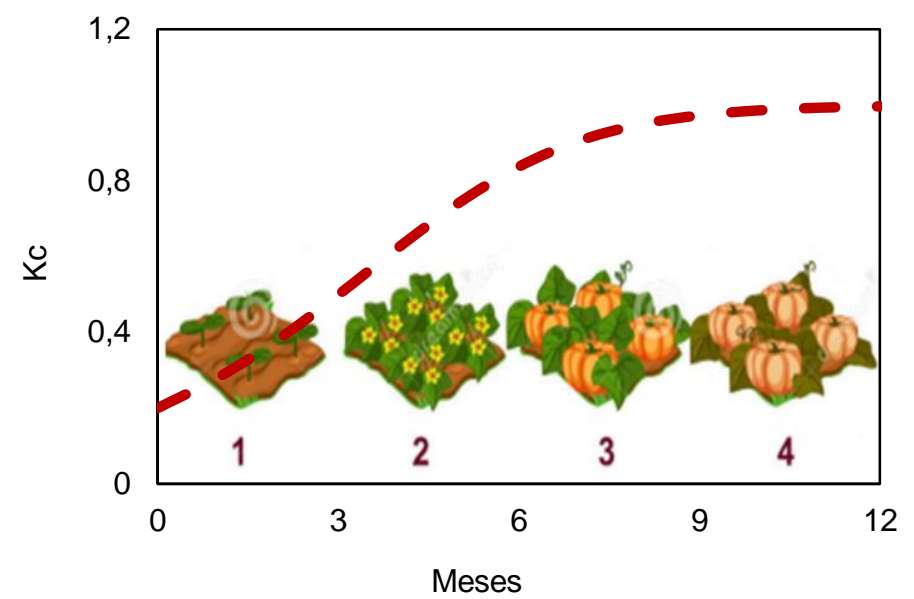

Fig. 4: Crecimiento promedio del cultivo de ahuyama teniendo en cuenta el coeficiente Kc y las semanas de crecimiento

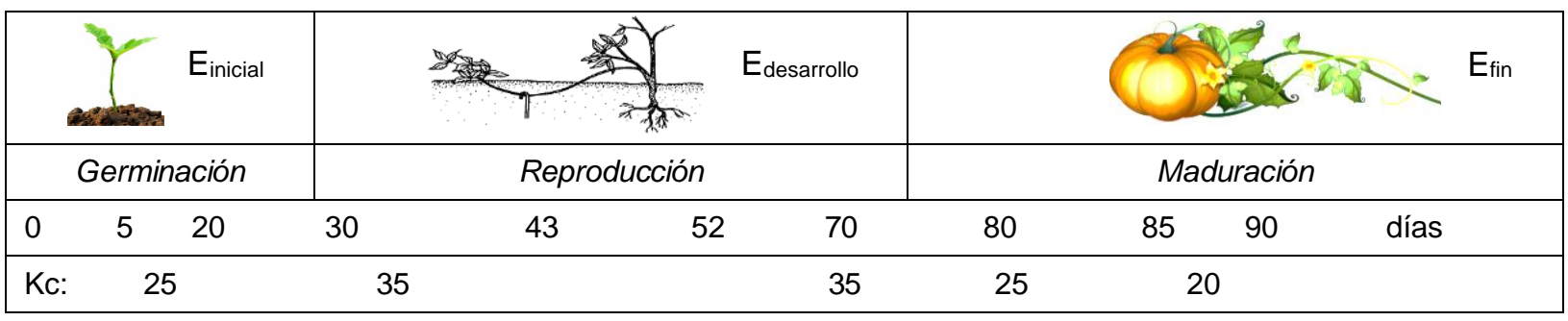

Fig. 5: Desarrollo de la planta de ahuyama en la zona de cultivo en Sincelejo, Colombia

Estos valores fueron calculados a través del modelo matemático desarrollado por la FAO (Allen, 2006) mostrado en (2). Donde $E T_{c}$ es la evapotranspiración del cultivo $(\mathrm{mm})$, el $\mathrm{K}_{\mathrm{c}}$ es el coeficiente de cultivo y $E T_{r}$ es la evapotranspiración de referencia $(\mathrm{mm})$, es decir que el consumo de agua en el cultivo va incrementando conforme el plantío va creciendo hasta alcanzar su punto máximo en la época de maduración, para luego descender hasta que el período de vida de las plantas llega a su fin.

$$
K_{c}=\frac{E T_{c}}{E T_{r}}
$$

\section{Estructura del suelo del cultivo}

La estructura del suelo donde se realizaron las pruebas del sistema de riego, obedece a la división de los cuatro horizontes mostrados en la Figura 6, estos son los descritos como las zonas A, B, C y D donde la zona $D$ pertenece a la estructura relacionada con la base del suelo o la roca madre, la zona $C$ es roca madre erosionada y generalmente no posee material orgánico, mientras que la zona $B$ es una franja de deposición (iluviación) caracterizada por facilitar la movilidad de la arcilla fina y, dependiendo del tipo de suelo (franco, arcilloso o la combinación de ambos) posee poca o muy poca materia orgánica, la zona A, es la región del suelo donde existe una mayor cantidad de material orgánico depositado (eluviones) y, por tanto, la que más intercambia elementos orgánicos con la flora y fauna existente. Por tanto, las zonas que más interesan para el cultivo de ahuyama son las zonas A y B, por poseer material orgánico suficiente, minerales y demás compuestos necesarios para el crecimiento del cultivo. Para el sistema de control de riego diseñado, la estructura del suelo ha sido modelada de manera que se utiliza como entrada útil el tipo de suelo, y con esto se define la capacidad de campo de este en las capas A y B, y su densidad aparente, a través de los modelos planteados en (Cohen-Manrique et al., 2018) para los tres tipos de suelos presentes en la zona de cultivo. Así mismo, fueron calculadas las pendientes de infiltración para establecer la rapidez con la que el agua se infiltra en el suelo, para ello se utilizaron modelos de regresión (Sihag et al., 2017). La zona E en la Figura 6, es la cobertura vegetal o tendido verde superficial, zona que no restringe el desarrollo radicular del cultivo. 


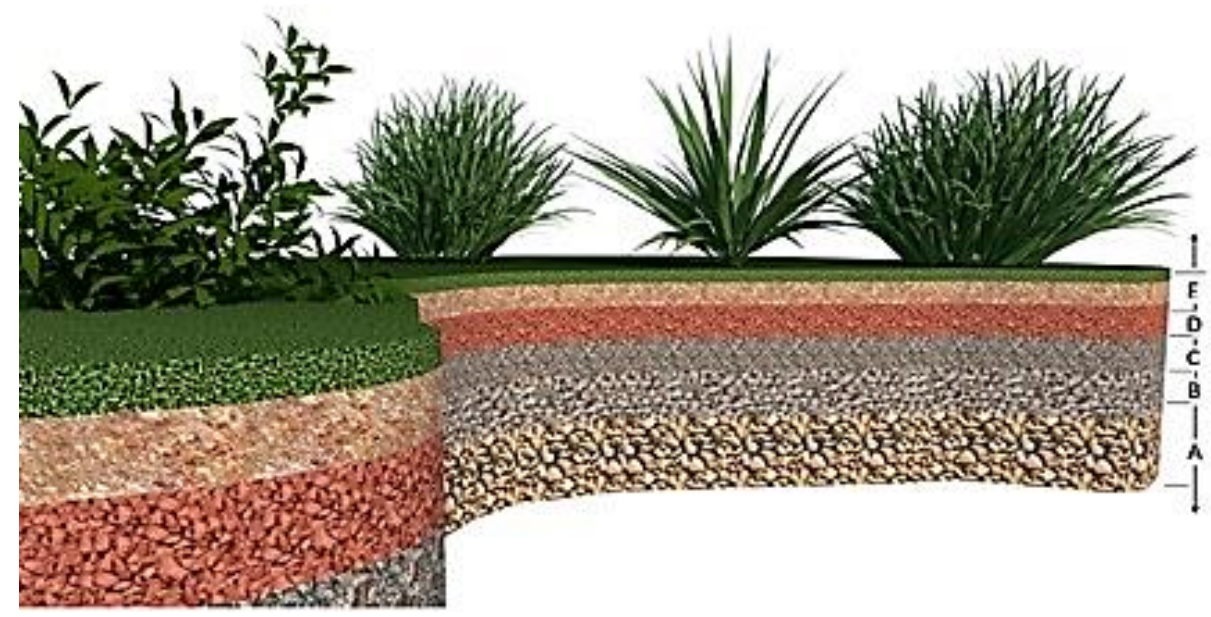

Fig. 6: Horizontes del suelo utilizado para el sistema de riego automático en el cultivo de ahuyama

\section{Sistema de cultivo basado en loT}

El sistema de cultivo, estuvo orientado hacia el manejo de los datos arrojados por la estructura de sensores inalámbricos a través de técnicas de big data (integración de datos) para la gestión de grandes cúmulos de información relacionados con el cultivo, el suelo, las variables ambientales, consumo energético del sistema, manejo de fertirriego, respuesta del mismo a lo largo del tiempo, entre otras variables; de modo que pudiese obtenerse y almacenarse la información suficiente para hacer modelamiento y simulación mediante técnicas predictivas (machine learning), para establecer el futuro del cultivo en cuanto a rendimiento, utilizando como parangón de esos resultados la experiencia de los agricultores de la región. En cuanto al almacenamiento de los datos, se utilizó un diseño de loT basado en la nube propuesto por (Farooq et al., 2019) para la agricultura de precisión. En este modelo, se aplicaron técnicas para el análisis y gestión de los datos del cultivo, mediante los sensores y dispositivos de monitoreo para la captura de información relevante en la toma de decisiones. Esta toma de decisiones es la implementada en el sistema operativo embebido (SOE) cargado en el procesador Intel de la tarjeta Galileo, y que se consulta a través de terminales computacionales o a través de dispositivos móviles. El sistema se diseñó e implementó sobre la base de cuatro capas que son Cloud Storage (módulos de control, consulta, computación y analítica de datos), Gateway (SOE, Gateway y los dispositivos móviles), Fog Computing (control de procesos y análisis de datos) y módulos de hardware (sensores, actuadores, entre otros). La capa de almacenamiento en la nube centralizó todos los datos relacionados con el cultivo.

\section{RESULTADOS Y DISCUSIÓN}

Uno de los resultados obtenidos son las gráficas de infiltración calculadas para los suelos de la región. En la figura 7a se muestran las tasas de infiltración y las láminas de agua acumuladas por el suelo a través del sistema de riego. La velocidad de infiltración del agua en el suelo es un parámetro muy importante a la hora de calcular el tiempo de riego en los cultivos, así como también la infiltración acumulada muestra la lámina de líquido que se llega a almacenar debido al mismo riego. Por esto se decidió modelar las tasas de infiltración en los diferentes tipos de suelo mediante modelos ajustados de Kostiakov-Lewis (Pérez-Leira et al., 2018). Se puede observar que la mayor velocidad de infiltración $(\mathrm{cm} / \mathrm{hr}$ ) es la producida en el suelo arenoso (línea color azul), suelo que de hecho se presenta en muy pocas locaciones del cultivo.

Las mayores extensiones del terreno utilizado para la siembra de la ahuyama estuvieron conformadas por suelos arcillosos, francos y franco arcillosos. La variable que mostró una mayor tasa de crecimiento en cuanto a la infiltración del agua fue el suelo franco (café) con una pendiente promedio de -4,86; seguida con el franco arcilloso con una pendiente promedio de $-4,29$ y el tipo de suelo que registró una velocidad menor fue el arcilloso con una tasa promedio de -3,93 (amarillo). Resultados comparables con (Acevedo et al., 2018) quienes al utilizar alternativas de diseño y manejo del riego por surcos evaluaron las velocidades de infiltración, aplicando modelos orientados a la dinámica de la humedad del suelo, esenciales para predecir las necesidades de agua de los cultivos mientras se adaptan a perturbaciones externas mediante redes neuronales. Así también (Aoki y Sereno, 2006) evaluaron en distintos terrenos la velocidad de infiltración utilizando simuladores de lluvias, con resultados análogos. Los parámetros utilizados como base para establecer la velocidad de infiltración fueron los propuestos por Van Genuchten para suelos típicos no saturados (Alanís, 2012). En cuanto a la figura 7b, se puede observar el promedio de la lámina acumulable de líquido a medida que se inicia el sistema de riego, de modo que se pueden identificar las cantidades de líquido que entrega el sistema y los tiempos de acción de este, con los que se define el consumo energético y sensibilidad de este. 
En la Figura 8 se muestra el trabajo realizado por el controlador PID sobre la electroválvula durante las 24 horas de trabajo del sistema de riego, es decir el porcentaje de apertura de la válvula a través del software diseñado en Matlab y que se implementa en el controlador. Es claro que en las horas que se presenta una mayor actividad, son las primeras cinco horas del día; el controlador distribuye la mayor cantidad de líquido al cultivo, llegando a abrir un valor porcentual máximo de $12.27 \%$ en las primeras 3 horas de funcionamiento, valores afines en cuanto al consumo energético y al trabajo realizado por la válvula para la correcta realización del riego mediante simulación (Gómez et al., 2018).

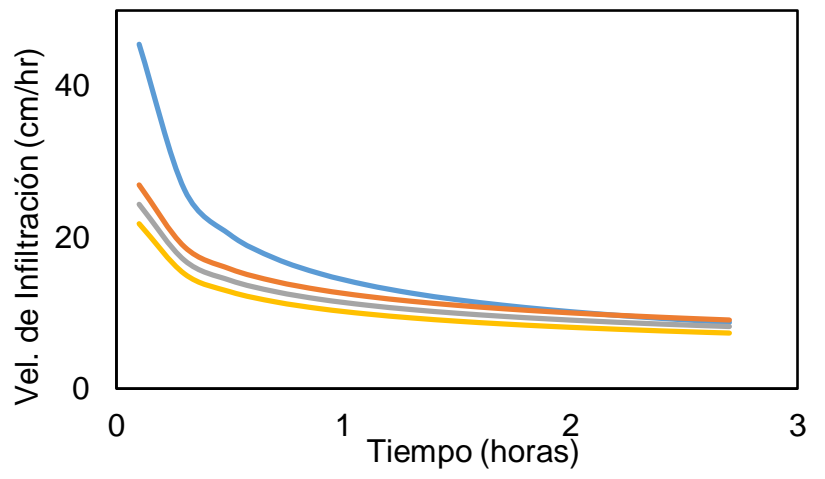

a.

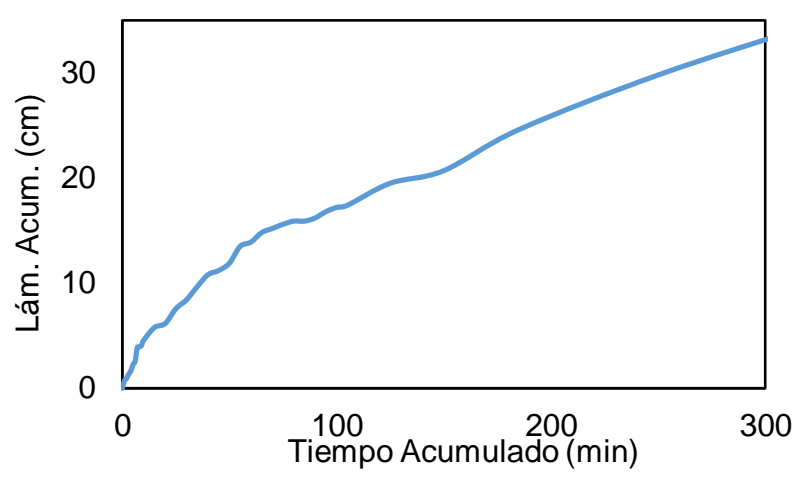

b.

Fig. 7: Comportamiento de la Infiltración en los suelos utilizados

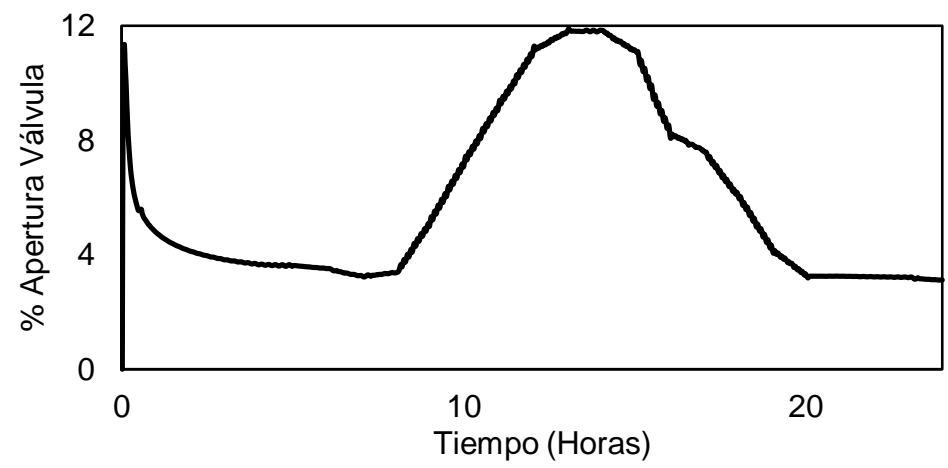

Fig. 8: Trabajo de apertura de la válvula debida al sistema de control PID

En cuanto al controlador, éste fue calibrado de modo que la variable error, que para éste diseño específico correspondía a la diferencia entre la variable humedad deseada en el suelo y humedad real en el cultivo, fuera lo más pequeña posible, y para ello se utilizaron los valores iniciales de la tabla 1 sugeridos por (Portnoy et al., 2019) y entregando como función de transferencia del controlador la ecuación 3, la cual generó valores de error por debajo del 2\%, tal como se aprecia en la tabla 2. Estos parámetros del controlador PID son semejantes a los planteados por (Blondin et al., 2018).

$$
C(S)=2+\frac{21}{200 S}+0.35 S=S^{2}+5.71 S+0.3675
$$

Tabla 1: Coeficientes definidos luego de la sintonización del controlador PID.

\begin{tabular}{|l|l|}
\hline \multicolumn{2}{|c|}{ Parámetros Controlador } \\
\hline $\mathrm{K}_{\mathrm{p}}$ & 2 \\
\hline $\mathrm{T}_{\mathrm{i}}$ & 4.7619 \\
\hline $\mathrm{T}_{\mathrm{d}}$ & 0.175 \\
\hline
\end{tabular}

Tabla 2: Valores promedio de la humedad real del cultivo y de la humedad calculada por la red WSAN en un día típico.

\begin{tabular}{|l|c|c|c|c|c|}
\hline Humedad Real & 0,04702313 & 0,18805493 & 0,46994921 & 0,84545712 & 1,07994574 \\
\hline Humedad Deseada & 0,04712232 & 0,18808150 & 0,46988519 & 0,84553949 & 1,07994799 \\
\hline Tiempo (Horas) & 1 & 4 & 10 & 18 & 23 \\
\hline
\end{tabular}


La Figura 9 muestra el comportamiento promedio diario del error entregado por el controlador sintonizado con los parámetros anteriormente descritos, es decir, la diferencia o distancia absoluta entre la variable que se tomó como referencia con el controlador PID y el valor de la variable leída del suelo. Valores que concuerdan con los establecidos en la tabla 2.

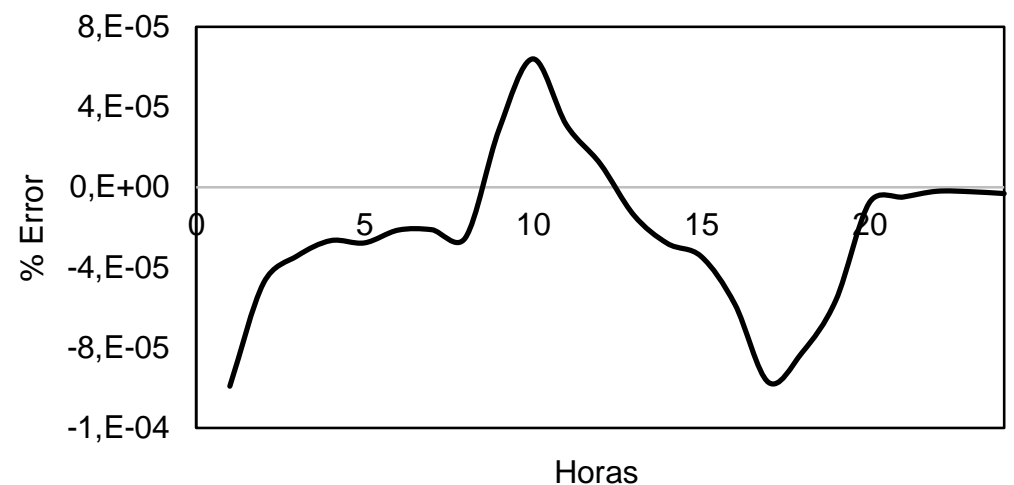

Fig. 9: Comportamiento de la variable error en el controlador PID ajustado

\section{DISCUSIÓN FINAL}

El internet de las cosas junto con los sistemas de control tradicionales como el PID, resultan ser herramientas válidas e importantes para la implementación de tecnologías relacionadas con la agricultura de precisión, sistemas de riego y modelamiento de variables naturales. El hecho de que luego de la implementación del modelado, del sistema de control y la infraestructura TI que soporta al internet de las cosas, los niveles de error en estado estable en la comparación de las variables de entrada (humedad deseada en el cultivo) y de salida (humedad real del cultivo) en el sistema de retroalimentación sean menores del $2 \%$, es una muestra clara de la utilidad de dichos sistemas y su potencia y rendimiento. Es decir que la implementación de sistemas tecnológicos basados en modelamiento, resultan ser óptimos como herramientas agroindustriales en regiones con alto impacto rural, se provee de mejores alternativas y posibilidades para gestionar los cultivos y optimizar los consumos de agua en los mismos. Así mismo, a medida en que se van haciendo más asequibles las tarjetas de control en placas de arduinos, y los sensores que componen la red de sensores (WSAN), se podrán contemplar dispositivos más potentes y a menores costos, orientados a fortalecer tecnológicamente los campos y el sector agrícola en el departamento de Sucre, tecnología de la cual actualmente adolece. Estos mismos resultados son consignados por (Ruíz-Martínez et al., 2019) quienes monitorearon y controlaron variables ambientales en cultivos de café a través de estas mismas plataformas, en Pueblo Tapao (Quindío, Colombia).

Así mismo, a través del presente estudio se encontró que, en el cultivo de ahuyama a campo abierto, en el cual se aplicó el sistema de riego PID, se utilizaron diferentes modelos matemáticos relacionados con los consumos de líquido por parte de la plantación tanto en el suelo, a través de la medición de la escorrentía, capilaridad, tasas de infiltración; así como también la tasa de consumo líquido debida a los factores atmosféricos tales como la evapotranspiración, la pluviosidad, entre otras. Mostrando un correcto acoplamiento mediante la herramienta software de simulación Simulink de Matlab y la tarjeta de procesamiento, de manera que este modelamiento se muestra de forma clara como una alternativa viable y eficiente ya que reduce el uso de recursos de operación para de este tipo de sistemas orientados a la agricultura de precisión. A continuación, se muestra el modelo en Matlab utilizado para la programación del controlador Galileo, encargado de capturar la información de las variables ambientales, que alimenta el modelo matemático de evapotranspiración, para de esta manera comparar el valor de la humedad deseada contra la humedad real del cultivo, que fue medida a través de la red sensórica instalada en el cultivo, y accionar la válvula a través del controlador PID sintonizado previamente.

El esquema de la figura 10 corresponde al modelo utilizado, donde se capturan los datos de las variables climatológicas que afectan al cultivo a través de la una red de sensores y actuadores inalámbricos WSAN que utiliza una topología de red en malla (mesh) conectada a un módulo zigbee que son alimentados con batería de litio y páneles solares para su autosustento energético. Los sensores enviaron información discontínua durante 15 días, como etapa de prueba, y durante este tiempo la información fue almacenada en la nube, para la aplicación de futuros modelos predictivos que coadyuven a la optimización de los recursos económicos, energéticos e hídricos. Así mismo, cabe mencionar, que el diseño mencionado tuvo algunos problemas de conectividad y de consumo de potencia, por tanto, para próximas aplicaciones de este tipo, se plantearían trabajos orientados hacía el diseño de algoritmos de optimización energética, de tiempos y que reduzcan costos de implementación. 


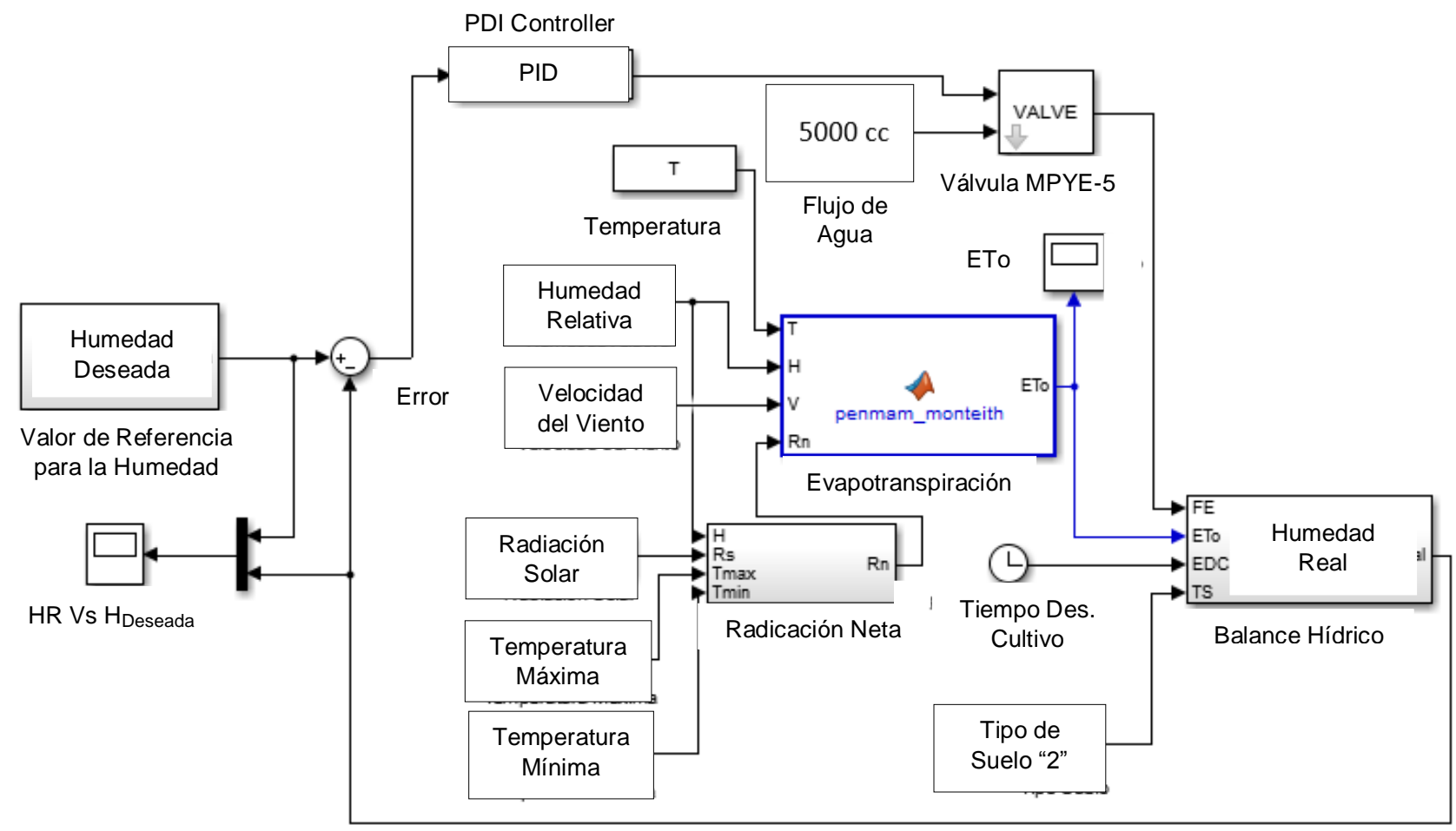

Fig. 10: Modelo esquemático del sistema de control PID para la apertura de la válvula MPYE-5

\section{CONCLUSIONES}

El controlador PID diseñado e implementado, demostró ser apropiado para el control y optimización del recurso hídrico para cultivos de ahuyama en la región Sabanas del departamento de Sucre, obteniendo que su rendimiento energético y precisión a la hora del riego arrojó muy buenos resultados, con porcentajes de error menores al $2 \%$, demandando bajos costos de implementación, y además, se aprecia que para éste tipo de aplicaciones agrícolas, los dispositivos utilizados no requieren altas velocidades de respuesta ni alta exactitud en su funcionamiento para ser eficientes, el diseño planteado resulta ser conveniente.

Así mismo, la implementación de la red de sensores y actuadores de bajo costo en campo, resultó ser una estrategia importante, conectándose entre ellos a través de una estructura de red en malla, con lo cual los tiempos de respuesta y consumo fueron acordes a la aplicación de riego requerida, es decir que respondieron conforme a lo solicitado por la estación central. Aunque, es importante destacar que el número de sensores de variables físicas del suelo, ubicados por hectárea (4) son relativamente bajos, así como la implementación de una estación meteorológica por cada 10 hectáreas aproximadamente, por lo cual, para mayor precisión se requeriría ampliar la colección de sensores en la red.

Además, por el hecho de utilizar modelos matemáticos probados para más del $70 \%$ de las variables relacionadas con el suelo, y que la totalidad de las variables meteorológicas son medidas a través de una estación en grandes áreas de terreno; los valores de implementación del diseño de control propuesto resultaron asequibles para el sector agrícola de la zona en la cual se aplicó de estudio, que tradicionalmente está liderada por campesinos con poco poder de inversión en la tecnificación de éste importante sector productivo. Lo que convierte a la alternativa propuesta en una opción viable y aplicable en grandes extensiones de terreno para la región.

\section{REFERENCIAS}

Acevedo, A. N., Sandoval I., y Salcedo, J., Desarrollo y productividad de ñame (Dioscorea trífida y Dioscorea esculenta) en diferentes condiciones hídricas. Acta agronómica 64(1), 30-35 (2015).

Aoki, A. M., y Serrano, R., Evaluación de la infiltración como indicador de calidad de suelo mediante un micro simulador de lluvias. Agriscientia 23(1), 23-31 (2006).

Alanís, A., Deformación volumétrica en suelos no saturados. Tesis de Maestría, Facultad de Ingeniería, Universidad Autónoma de Querétaro. Extraído de: http://ri.uaq.mx/browse, México, (2012).

Allen, R. G., Evapotranspiración del cultivo: guías para la determinación de los requerimientos de agua de los cultivos. Food \& Agriculture Org Vol. 56 (2006). 
Blondin, M. J., Sanchis, J., y otros dos autores, New optimal controller tuning method for an AVR system using a simplified Ant Colony Optimization with a new constrained Nelder-Mead algorithm. Applied soft computing 62, 216-229 (2018).

Castellaro, G., y Squella, F., Modelo simple de simulación para la estimación del crecimiento, fenología y balance hídrico de praderas anuales de clima Mediterráneo. https://doi.org/10.4067/S0365-28072006000300006. Agricultura técnica 66(3), 271-282 (2006).

Cohen-Manrique, C., Rodríguez-Manrique, J., y otros dos autores, Diffuse irrigation systems for the production of watermelon in the Sabanas subregion. https://doi.org/10.15446/dyna.v86n208.72966. Dyna 86(208), $243-250$ (2019).

Cohen-Manrique, C., Rodríguez-Manrique, J., y Salgado-Ordosgoitia, R., Modelado del Microclima de un Cultivo de Sandía (Citrullus lanatus) en la Sub-región Sabana del Departamento de Sucre. https://doi.org/10.4067/S071807642018000500335. Información tecnológica 29(5), 335-344 (2018).

Çelekli, A., Bozkuş, B., y Bozkurt, H., Development of a new adsorbent from pumpkin husk by KOH-modification to remove copper ions. https://doi.org/10.1007/s11356-017-1160-2. Environmental Science and Pollution Research 26(12), 1151411523 (2019).

Domínguez-Pérez, F., y Mercado-Fernández, T., Potential infiltration and morphometry in the Arroyo Grande basin, Sucre Colombia. Https://doi.org/10.17533/udea.redin.20191043. Revista Facultad de Ingeniería Universidad de Antioquia (96), 21-31 (2020).

Farooq, M. S., Riaz, S., y otros tres autores, A Survey on the Role of IoT in Agriculture for the Implementation of Smart Farming. https://doi.org/10.1109/ACCESS.2019.2949703. IEEE Access 7, 156237-156271 (2019).

Marsiglia Fuentes, R. M., Torregroza Fuentes, E., y otros dos autores, Application of geographic information systems for characterization of preharvest and postharvest factors of squash (cucurbita sp.) in Bolívar Department, Colombia. https://doi.org/10.17485/ijst/2018/v11i9/117914. Indian Journal of Science and Technology 11(9), 1-10 (2018).

García, L., Parra, L., y otros tres autores, IoT-Based Smart Irrigation Systems: An Overview on the Recent Trends on Sensors and loT Systems for Irrigation in Precision Agriculture. https://doi.org/10.3390/s20041042. Sensors 20(4), 1042 (2020).

Gómez, J. C., Rodríguez, D., y Díaz, J., Irrigation control system with monitoring functions for hydroponic crops based on the demand tray method. Pistas educativa, 40(130), 1-15 (2018).

González, A., Viloria, O., y otros cuatro autores, Isohyetal Maps of Daily Maximum Rainfall for Different Return Periods for the Colombian Caribbean Region. https://doi.org/10.3390/w11020358. Water 11(2), 358 (2019).

Hernández, G., Modelamiento eco-hidrológico de la humedad del suelo en el valle del río Cauca. Doctoral dissertation. Universidad Nacional de Colombia. Colombia, (2010).

Kirkham, M., Principles of soil and plant water relations. 2a edición. Academic Press. USA. (2014).

Liu, W., \& Kwaku, A., Enhancing product innovation performance in a dysfunctional competitive environment: The roles of competitive strategies and market-based assets. https://doi.org/10.1016/j.indmarman.2018.01.006. Industrial Marketing Management 73, 7-20 (2018).

Lozano-Rivas, W. A., Clima, hidrología y meteorología: Para ciencias ambientales e ingeniería. Universidad Piloto de Colombia. Colombia, (2018).

Orozco, A., Correa, E., y otros cuatro autores, Análisis de un sistema productivo agrícola en el Caribe: tecnología de producción, patrón de costos e indicadores económicos de la producción de ahuyama. https://doi.org/10.14482/ecoca.23.3033. Revista de Economía del Caribe (23), 46-69 (2019).

Pérez-Leira, R., Cabrera-Estupiñán, E., y otros cuatro autores, Irrigation Regime for Crops in Manabí, Ecuador: Edaphic Study. Revista Ciencias Técnicas Agropecuarias, 27(4), 1-8 (2018).

Pinilla, N. S., El cultivo de ahuyama (Cucurbita moschata) híbrida bárbara un modelo demostrativo y productivo a corto plazo en la vereda la Unión del municipio de Puerto Lleras Meta. https://ciencia.lasalle.edu.co/ingenieria_agronomica/73. Tesis de pregrado, Universidad de la Salle (2017).

Portnoy, I. D., Verdeza, A., y otros dos autores, Ecuaciones de Sintonía para Controladores PID Usando la Minimización del Error y de la Varianza de la Señal del Controlador como la Función Objetivo. https://doi.org/10.4067/S071807642019000100049. Información Tecnológica 30(1), 49-62 (2019).

Rodríguez R, R., Valdés R, M., y Ortiz G, S., Características Agronómicas y Calidad Nutricional de los Frutos y Semillas de Zapallo Cucúrbita. https://doi.org/10.24188/recia.v10.n1.2018.636. RECIA 10(1), 86-97 (2018).

Ruíz-Martínez, W., Díaz-Gutiérrez, Y., y otros dos autores, Application of the Internet of Things through a Network of Wireless Sensors in a Coffee Crop for Monitoring and Control its Environmental Variables. https://doi.org/10.22430/22565337.1485. Tecnológicas 22(46), 101-116 (2019).

Sihag, P., Neil, T., y Subodh, R., Modelling of infiltration of sandy soil using gaussian process regression. https://doi.org/10.1007/s40808-017-0357-1. Modeling Earth Systems and Environment 3(3), 1091-1100 (2017). 